\title{
Simplification and Identification of Discrete Transfer Function via Step-response Matching
}

by c. G. CHUNG

Department of Computer Science, Chiao Tung University, Hsinchu, Taiwan, Republic of China

K. W. HAN

Chung-Shan Institute of Science and Technology, Lung-Tan, Taiwan, Republic of China

and $\mathrm{H}$. H. YEH

Department of Electrical Engineering, University of Kentucky, Lexington, KY 40506, U.S.A.

ABSTRACT: A computer-aided method for simplification and identification of linear discrete systems via step-response matching is presented. Golub's algorithm for solving least-squares problem is used to find the optimum coefficients of the reduced model. The advantages of this method are (1) for model reduction, both the time response and frequency response within the bandwidth region of the reduced model are very close to those of the original system; and (2) for system identification, the identified model is very close to the original system. In the illustrative examples considered in this paper the results of the proposed method appear to be better than those of other methods in the current literature.

\section{Introduction}

Since the analysis of higher-order systems is tedious and costly, the problem of approximating a high-order system by a low-order model is important in the analysis and design of complex control systems. In this paper a computer-aided approach for model reduction and system identification of discrete systems via step-response matching is presented.

The problem of model reduction of linear time-invariant causal discrete systems can be defined as finding a low-order model with transfer function

$$
H(z)=\frac{\mathrm{d}_{m} z^{m}+\mathrm{d}_{m-1} z^{m-1}+\ldots+\mathrm{d}_{1} z+\mathrm{d}_{n}}{z^{n}+c_{n-1} z^{n-1}+\ldots+c_{z}+c_{0}}, \quad n>m
$$

to approximate a high-order system with transfer function

$$
G(z)=\frac{b_{M} z^{M}+b_{M-1} z^{M-1}+\ldots+b_{1} z+b_{0}}{z^{N}+a_{N-1} z^{N-1}+\ldots+a_{1} z+a_{0}}, \quad N>M \text { and } N>n
$$


so that the performance of the model $H(z)$ is very close to that of the original system $G(z)$. A number of methods for the reduction of discrete transfer functions have been presented in the current literature (1-7). Shih and $\mathrm{Wu}(2)$ applied the continued fraction method to discrete transfer functions. The disadvantage of this method is that the initial output response of the reduced model may not be zero, although that of the original system is zero. Shamash (3) proposed the method of continued fraction expansion about $z=1$ or $z=\infty$ to reduce the order of discrete transfer functions. The disadvantage is that the resulted model may not be stable although the original system is stable. Chuang (5) used linear transformation and homographic transformation to transform the original transfer function into the $v$-planc, and then applicd Pade' approximation to find the reduced-model. Although the reduced model found by this method has no stability problem, the performance seems not good. Shieh et al. (6) used the method of moment matching and retaining dominant eigenvalues to reduce the order of the discrete transfer functions. The accuracy of this method seems better than the others. Recently, a reduction method based on the stability-equations has been presented by Chen and Han (7). In this method the original transfer function is transformed into stability equations, then the roots of stability-equations having large magnitudes are discarded. Finally the reduced models are constructed based on the reduced stability-equations.

The concept of using step-response matching to find the reduced model of the original system was first proposed by Edgar (8). Since all poles of the reduced model should be real in Edgar's method this would be difficult to apply to discrete systems.

If the original discrete system is stable, its step response will reach steady state after a finite time. Since the number of sampling instants in this finite time can be assumed constant, the square errors between the response at the sampling instants of the original system and that of the reduced model can be used as the criterion of the matching between the original system and the reduced model. In this paper, Golub's algorithm is applied in order to find the coefficients of the reduced model because it can give the true minimum point of the square errors. If the step response of the reduced model matches that of the original system very closely, the reduced model will be stable, so the stability problem can be relaxed. In addition, the poles of the reduced model can be real and complex, hence it has less restriction than Edgar's method.

\section{Review of Golub's Algorithm}

Golub's algorithm is a numerical algorithm for solving least-squares problems in a highly accurate manner. Breen et al. (9) and Kao (10) have applied this algorithm in circuit analysis and optimization very successfully. The concept of this algorithm for least-squares problems is described briefly as follows.

Consider the system of linear equations

$$
\mathbf{A X}=\mathbf{B}
$$


where $\mathbf{A}$ is a given $(n \times m)$ real matrix of rank $m, \mathbf{B}$ is a given $(n \times 1)$ real column vector, and $\mathbf{X}$ is an unknown $(m \times 1)$ real column vector. Equation (3) has a unique solution if $n=m$. Suppose $n>m$, and we wish to find a vector $\hat{\mathbf{X}}$ such that

$$
\|\mathbf{B}-\mathbf{A} \hat{\mathbf{X}}\|=\min
$$

where $\|.$.$\| indicates the euclidean norm. Then we may choose an orthogonal$ matrix $\mathbf{Q}$ such that

$$
\mathbf{Q A}=\mathbf{R}=\left[\begin{array}{c}
\tilde{\mathbf{R}} \\
\cdots \\
\mathbf{0}
\end{array}\right] \quad \begin{aligned}
& m \times m \\
& (n-m) \times m
\end{aligned}
$$

and $\mathbf{R}$ is an upper triangular matrix as

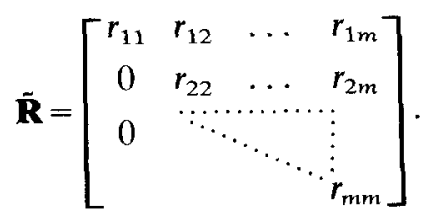

Then

$$
\|\mathbf{B}-\mathbf{A X}\|=\|\mathbf{Q B}-\mathbf{Q A X}\|=\|\mathbf{C}-\mathbf{R X}\|
$$

where $\mathbf{C}=\mathbf{Q B}$. And equation (7) can be rewritten as

$$
\begin{aligned}
\|\mathbf{B}-\mathbf{A X X}\|= & {\left[\left(c_{1}-r_{11} x_{1}-r_{12} x_{2} \ldots-r_{1 m} x_{m}\right)^{2}+\left(c_{2}-r_{22} x_{2}-\ldots-r_{2 m} x_{m}\right)^{2}\right.} \\
& \left.+\ldots+\left(c_{m}-r_{m m} x_{m}\right)^{2}+c_{m+1}^{2}+c_{m+2}^{2}+\ldots+c_{n}^{2}\right]^{\frac{1}{2}} .
\end{aligned}
$$

Thus $\|\mathbf{B}-\mathbf{A X}\|$ is minimized when

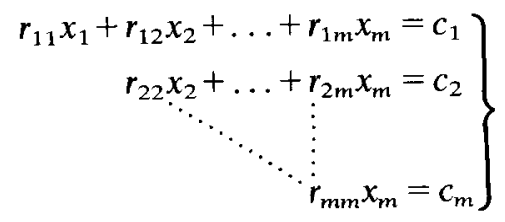

and the minimized value is

$$
\|\mathbf{B}-\mathbf{A X X}\|=\left(c_{m+1}^{2}+c_{m+2}^{2}+\ldots+c_{n}^{2}\right)^{\frac{1}{2}} .
$$

The decomposition of (5) can be realized by the Householder transformations. The detail of decomposition can be found in Kao (10), Householder (11), and Wilkinson (12).

From the above analysis, it can be seen that Golub's algorithm is suitable and can be applied to the least-squares errors problems. Assume the cost 


\section{G. Chung, K. W. Han and H. H. Yeh}

function $F$ is defined as

$$
F(\mathbf{d})=\sum_{i=1}^{n} e_{i}^{2}\left(\mathbf{d}, t_{i}\right)
$$

where $t_{\mathbf{i}}$ represents the independent variable sampled in $T$, $\mathbf{d}$ is a dependent variable vector with $m$ elements, $e_{i}$ is the error function at $t_{i}$, and $n$ is the total number of sampled points in $T$. The purpose is to find the optimum parameter vector such that the cost function $F$ is minimized. The partial derivative of $e_{i}(d)$ with respect to $\mathbf{d}$ is $\mathbf{e}_{i}^{\prime}(\mathbf{d})=\left(\partial e_{i}(\mathbf{d})\right) /(\partial \mathbf{d})$. And Taylor's series of $e_{i}(\mathbf{d})$ is

$$
e_{i}(\mathbf{d}+\Delta \mathbf{d})=e_{i}(\mathbf{d})+\mathbf{e}_{i}^{\prime}(\mathbf{d}) \Delta \mathbf{d}+\frac{1}{2 !} \mathbf{e}_{i}^{\prime \prime}(\mathbf{d})(\Delta \mathbf{d})^{2}+\ldots
$$

If $\Delta \mathbf{d}$ is very small, the second-order and higher-order terms can be neglected, then we get

$$
e_{i}(\mathbf{d}+\Delta \mathbf{d}) \cong e_{i}(\mathbf{d})+\mathbf{e}_{i}^{\prime}(\mathbf{d}) \Delta \mathbf{d} .
$$

In order to minimize the cost function $F(\mathbf{d})$, the direction vector $\Delta \mathbf{d}$ should be in the direction so that $e_{i}(\mathbf{d}+\Delta \mathbf{d})=0$. So $\Delta \mathbf{d}$ can be determined by the equation

$$
e_{i}(\mathbf{d}+\Delta \mathbf{d})=e_{i}(\mathbf{d})+\mathbf{e}_{i}^{\prime}(\mathbf{d}) \Delta \mathbf{d}=0 \quad i=1,2, \ldots, n
$$

or

$$
\mathbf{e}_{i}^{\prime}(\mathbf{d}) \Delta \mathbf{d}=-e_{i}(\mathbf{d})
$$

which can be rewritten as

$$
\left[\begin{array}{cccc}
\frac{\partial e_{1}}{\partial d_{1}} & \frac{\partial e_{1}}{\partial d_{2}} & \cdots & \frac{\partial e_{1}}{\partial d_{m}} \\
\frac{\partial e_{2}}{\partial d_{1}} & \frac{\partial e_{2}}{\partial d_{2}} & \cdots & \frac{\partial e_{2}}{\partial d_{m}} \\
\cdots & \cdots & \cdots & \ldots \\
\frac{\partial e_{n}}{\partial d_{1}} & \frac{\partial e_{n}}{\partial d_{2}} & \cdots & \frac{\partial e_{n}}{\partial d_{m}}
\end{array}\right]\left[\begin{array}{c}
\Delta d_{1} \\
\Delta d_{2} \\
\cdot \\
\cdot \\
\cdot \\
\Delta d_{m}
\end{array}\right]=\left[\begin{array}{c}
-e_{1} \\
-e_{2} \\
\cdot \\
\cdot \\
\cdot \\
-e_{n}
\end{array}\right]
$$

Since $n>m$, we can apply Golub's algorithm to solve (15) and find the value $\Delta$ d.

However, due to the approximation made in (14) and the fact that $\Delta d$ is only a least square solution, the new parameter vector $\mathbf{d}+\Delta \mathbf{d}$ does not, in general, minimize the cost function $F(\mathbf{d})$. The direction of $\Delta \mathbf{d}$ can only indicate that if $\mathbf{d}$ is changed infinitesimally small in the direction of $\Delta d$ the cost function $F(d)$ will be reduced. That means

$$
F(\mathbf{d}+\alpha \Delta \mathbf{d})<F(\mathbf{d})
$$

where $\alpha$ is called step size and $0<\alpha \leq 1$. The cost function $F(\mathbf{d})$ vs $\alpha$ can be plotted as shown in Fig. 1. The value of $\alpha$ should be chosen properly, otherwise, $F(\mathbf{d}+\alpha \Delta \mathbf{d})$ wil be greater than $F(\mathbf{d})$ or the convergent rate is too 


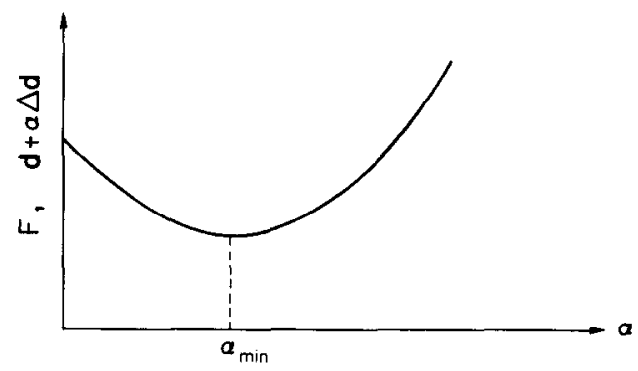

FIG. 1. The cost function $F(\mathbf{d}+\alpha \Delta \mathbf{d})$ vs the step size $\alpha$.

slow. There are many algorithms which can be used to find the optimum $\alpha$ (10), but we will not describe the details here.

Given the initial $\mathbf{d}$ as $\mathbf{d}^{0}$, we may evaluate the value of the cost function $F(\mathbf{d})$ as $F^{\circ}$. Using the above procedures to find $\Delta \mathbf{d}$ and $\alpha$, we get the new value of $\mathbf{d}$ as $\mathbf{d}^{1}$ and have $F\left(\mathbf{d}^{0}\right)>F\left(\mathbf{d}^{1}\right)$. Repeat the above procedures until $F(\mathbf{d}+\alpha \Delta \mathbf{d})$ or the difference between $F(\mathbf{d}+\boldsymbol{\alpha} \Delta \mathbf{d})$ and $F(\mathbf{d})$ is within the desired range, then the optimum parameter vector can be obtained. All the above procedures can be programmed in FORTRAN IV or other high-level languages, and the results can be easily obtained.

\section{Description of the Reduction Method}

Consider the transfer function in (2), which can be written in a non-recursive form as

$$
G(z)-r_{0}+r_{1} z^{-1}+r_{2} z^{-2}+\ldots
$$

where $r_{i}$ is equivalent to $g(i T)$, which is the impulse response of this discretetime system at the sampling instant $i T$. Since the $z$-transform of the unit-step function is

$$
R(z)=\frac{1}{1-z^{-1}}=1+z^{-1}+z^{-2}+z^{-3}+\ldots
$$

the unit-step response of $G(z)$ is

$$
\begin{aligned}
Y(z) & =G(z) R(z) \\
& =\left(r_{0}+r_{1} z^{-1}+r_{2} z^{-2}+\ldots\right)\left(1+z^{-1}+z^{-2}+\ldots\right) \\
& =r_{0}+\left(r_{0}+r_{1}\right) z^{-1}+\left(r_{0}+r_{1}+r_{2}\right) z^{-2}+\ldots \\
& =\beta_{0}+\beta_{1} z^{-1}+\beta_{2} z^{-2}+\ldots
\end{aligned}
$$

where $\beta_{i}=\sum_{k=0}^{i} r_{k}$, and $\beta_{i}$ is the unit-step response of this discrete system at sampling instant $i T$. In (2) substituting $z$ by $\mathrm{e}^{\mathrm{j} \omega \mathrm{T}}$, the frequency response of $G(z)$ is resulted as:

$$
G\left(\mathrm{e}^{j \omega T}\right)=\frac{b_{\mathrm{M}} \mathrm{e}^{j M \omega T}+b_{\mathrm{M}-1} \mathrm{e}^{j(M-1) \omega T}+\ldots+b_{1} \mathrm{e}^{j \omega T}+b_{0}}{\mathrm{e}^{j N \omega T}+a_{N-1} \mathrm{e}^{j(N-1) \omega T}+\ldots+a_{1} \mathrm{e}^{j \omega T}+a_{0}}
$$


If the system $G(z)$ is stable, the steady-state unit-step response will be equal to the corresponding frequency response at $\omega=0$, i.e.

$$
\left.G\left(\mathrm{e}^{\mathrm{j} \omega \mathrm{T}}\right)\right|_{\omega=0}=\frac{b_{M}+b_{M-1}+\ldots+b_{1}+b_{0}}{1+a_{N-1}+a_{N-2}+\ldots+a_{1}+a_{0}} .
$$

Similarly, if the reduced model $H(z)$ is stable, the frequency response of the reduced model, $H\left(\mathrm{e}^{\mathrm{i} \omega \mathrm{T}}\right)$, at $\omega=0$ can be expressed by

$$
\left.H\left(\mathrm{e}^{j \omega T}\right)\right|_{\omega=0}=\frac{d_{m}+d_{m-1}+\ldots+d_{1}+d_{0}}{1+c_{n-1}+c_{n-2}+\ldots+c_{1}+c_{0}} .
$$

If the steady-state unit-step response of the reduced model is equal to that of the original system, then

Hence

$$
\begin{aligned}
\left.G\left(\mathrm{e}^{j \omega T}\right)\right|_{\omega=0} & =\left.H\left(\mathrm{e}^{\mathrm{j} \omega \mathrm{T}}\right)\right|_{\omega=0} \\
& =\frac{d_{m}+d_{m-1}+\ldots+d_{1}+d_{0}}{1-c_{n-1}+c_{n-2}+\ldots+c_{1}+c_{0}} .
\end{aligned}
$$

$$
d_{0}=G\left(\mathrm{e}^{j O T}\right)\left[1+\sum_{i=0}^{n-1} c_{i}\right]-\sum_{i=1}^{m} d_{i}
$$

Suppose the unit step response of $G(z)$ and $H(z)$ are denoted as $y_{1}(i T)$ and $y_{2}(i T)$ and they reach steady state at the sampling instant $K T$ (where $K$ is a positive integer). For simplicity of notation let us define $\mathbf{C}=\left(c_{0}, c_{1}, \ldots, c_{n-1}\right)$, and $\mathbf{D}=\left(d_{1}, d_{2}, \ldots, d_{m}\right)$. Now the closeness of the reduced model and the original system can be defined by the cost function

$$
E(\mathbf{C}, \mathbf{D})=\sum_{i=1}^{K} e_{i}^{2}=\sum_{i=1}^{K}\left[y_{1}(i T)-y_{2}(i T, \mathbf{C}, \mathbf{D})\right]
$$

which is equivalent to the sum of square-errors between the step response of the original system, $y_{1}(i T)$, and that of the reduced model, $y_{2}(i T)$, for $i$ from 1 to $K$. Then the coefficients of the reduced model (i.e. the coefticient vectors $\mathbf{C}$ and $\mathbf{D})$ can be found by minimizing the cost function.

Thus the initial values of coefficient vectors $\mathbf{C}$ and $\mathbf{D}$ of the reduced model as $\mathbf{C}^{0}$ and $\mathbf{D}^{\mathbf{0}}$ will make the resulting system stable. We may use (20) to find the step-responses of $G(z)$ and $H(z)$. Since $K$ is usually chosen very large (i.e., $K \geq 15)$ and the order of $H(z)$ should be as low as possible, hence $K$ is always greater than $n+m$, and the process described in Section II can be applied iteratively to find the optimum coefficients of the reduced model.

Since the sampling frequency is defined as $\omega_{s}=2 \pi / T$, from (21) the frequency response of $G(z)$ can be rewritten as

$$
\begin{aligned}
G\left(\mathrm{e}^{j \omega T}\right)= & G\left(\mathrm{e}^{j 2 \pi\left(\omega / \omega_{s}\right)}\right)=r_{0}+r_{1} \mathrm{e}^{-j 2 \pi\left(\omega / \omega_{s}\right)}+r_{2} \mathrm{e}^{-j 2 \pi\left(2 \omega / \omega_{s}\right)}+\ldots \\
= & {\left[r_{0}+r_{1} \cos \left(2 \pi \frac{\omega}{\omega_{s}}\right)+r_{2} \cos \left(2 \pi \frac{2 \omega}{\omega_{s}}\right)+\ldots\right] } \\
& -j\left[r_{1} \sin \left(2 \pi \frac{\omega}{\omega_{s}}\right)+r_{2} \sin \left(2 \pi \frac{2 \omega}{\omega_{s}}\right)+\ldots\right]
\end{aligned}
$$


At low frequency, $\cos (\omega T)$ approaches 1 and $\sin (\omega T)$ approaches 0 , so that the real part of $G\left(\mathrm{e}^{j \omega T}\right)$ dominates the frequency response and the value is approximately equal to $\sum_{i=0}^{\infty} r_{i}$ which is equivalent to the unit-step response of $G(z)$. Thus we find from the illustrative examples that the frequency response within bandwidth region of the reduced model is very close to that of the original system.

If two systems are equivalent, they will have the same unit-step response. Therefore this method can be used to identify the parameters of linear time-invariant discrete systems.

It should be noted that, since the proposed method has less restrictions than the other methods in the current literature, the accuracy of the proposed method is high. This will be shown by the following examples.

Example 1. Consider the transfer function used by Shih et al. $(\mathbf{2}, \mathbf{6}, \mathbf{7})$ :

$G(z)$

$=\frac{280.333 z^{7}+186 z^{6}-35 z^{5}+25.333 z^{4}-86 z^{3}-43.666 z^{2}+7.333 z-1}{666 z^{8}-280.333 z^{7}-186 z^{6}+35 z^{5}-25.333 z^{4}+86 z^{3}+43.666 z^{2}-7.333 z+1}$

The sampling period is $T=\sqrt{ } 0.5$. Suppose the second-order model is chosen as

$$
H(z)=\frac{0.5 z+0.15}{z^{2}-0.8 z+0.15}
$$

and the step responses of $G(z)$ and $H(z)$ at first 30 sampling instants are chosen for comparison. Using the process described in Section II, after 8 iterations the reduced model can be obtained as

$$
H_{2}(z)=\frac{0.460997-0.303206}{z^{2}-1.530156 z+0.687127} .
$$

By the same process, the third-order reduced model is

$$
H_{3}(z)=\frac{0.42604 z^{2}-0.304414 z+0.000989}{z^{3}-1.722563 z^{2}+0.991368 z-0.146425} .
$$

For comparison, the reduced models obtained by Shih et al. (6) and by Chen $e t$ al. (7) are listed as follows:

$$
\begin{gathered}
H(z)=\frac{0.4981 z-0.34194}{z^{2}-1.50189 z+0.65805} \quad \text { (by Shih) } \\
F_{2}(z)=\frac{10 z-8}{25.1575 z^{2}-40.315 z+17.158} \quad \text { (by Chen) }
\end{gathered}
$$

and

$$
F_{3}(z)=\frac{15.948 z^{2}-20.895 z+6.948}{35.944 z^{3}-77.674 z^{2}+58.516 z-14.786} \quad \text { (by Chen). }
$$


C. G. Chung, K. W. Han and H. H. Yeh

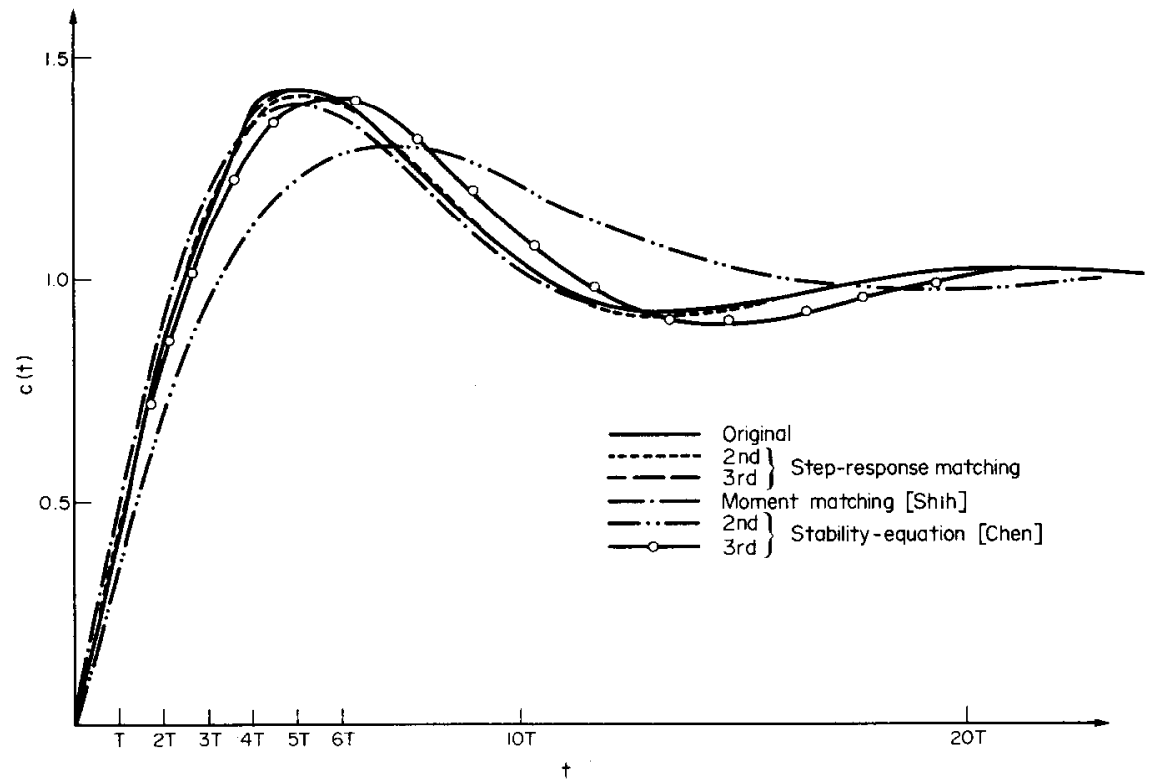

Fig. 2. Step responses of example 1.

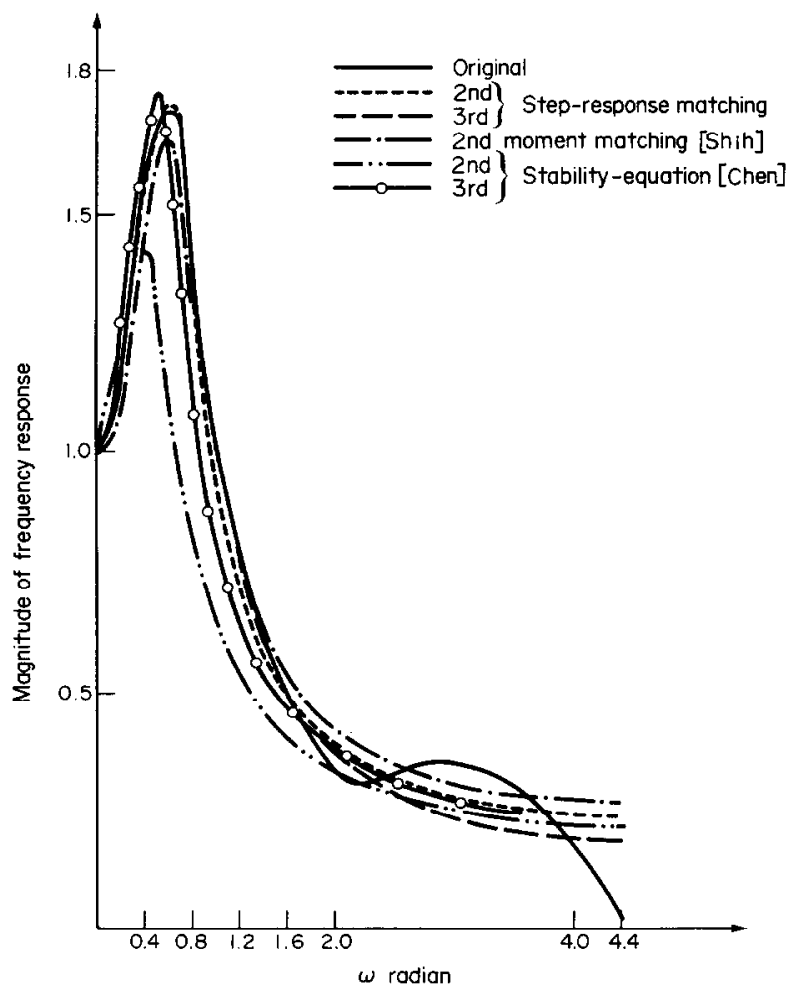

FIG. 3. Gain plots of frequency responses of example 1. 
The step responses and frequency responses of all these reduced models are shown in Figs. 2 and 3, respectively. It can be seen that in both cases the reduced models based on the proposed method can give better results. Note that the frequency response within the bandwidth region of the reduced method obtained by the proposed method is very close to that of the original system.

Example 2. Consider the system shown in Fig. 4 (13). The sampling period T is $0.15 \mathrm{sec}$, and the closed-loop transfer function is

$$
G(z)=\frac{0.4240368 z^{3}+0.0125156 z^{2}-0.3118169 z+0.570404}{z^{4}-1.0966632 z^{3}-0.1434224 z^{2}+0.6953299 z-0.2734684} .
$$

Assume the step response of the first 30 sampling instants are selected for the purpose of step-response matching. The 2 nd and 3 rd order reduced models are obtained as

$$
H_{2}(z)=\frac{0.4606312-0.1752814}{z^{2}-1.2995513 z+0.5849011}
$$

and

$$
H_{3}(z)=\frac{0.4307344 z^{2}-0.2896451 z-0.0009796}{z^{3}-1.7335551 z^{2}+1.1429416 z-0.2692771} .
$$

The cost function of the 2 nd and 3 rd order reduced models are approximately equal to $4.5 \times 10^{-3}$ and $5.4 \times 10^{-4}$, respectively. If the cost function obtained is not satisfied, we may assume a 4 th order model based upon the optimum $3 \mathrm{rd}$ model as

$$
H_{4}^{0}(z)=\frac{0.4307344 z^{3}-0.2896451 z^{2}-0.0009796 z}{z^{4}-1.7335551 z^{3}+1.1429416 z^{2}-0.2692771 z} .
$$

After 8 iterations, the cost function is less than $10^{-10}$ and the reduced model is

$$
H_{4}(z)=\frac{0.4240368 z^{3}-0.0125180 z^{2}-0.3118165 z-0.0570391}{z^{4}-1.0966577 z^{3}-0.1434277 z^{2}+0.6953296 z-0.2734668} .
$$

Note that all the coefficients of (39) are almost the same as those of (35). Therefore, the proposed process can be applied to discrete system identification via step-response matching.

For comparison, the step responses and frequency responses of the original system and the reduced models of all the methods considered are shown in

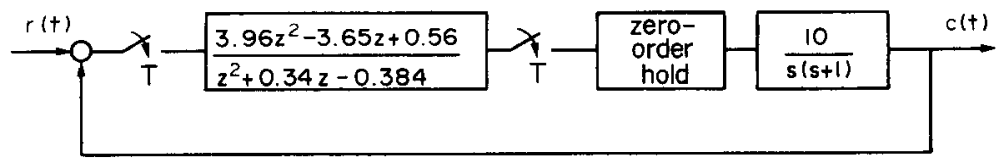

FIG. 4. A sampled-data control system. 
C. G. Chung, K. W. Han and H. H. Yeh

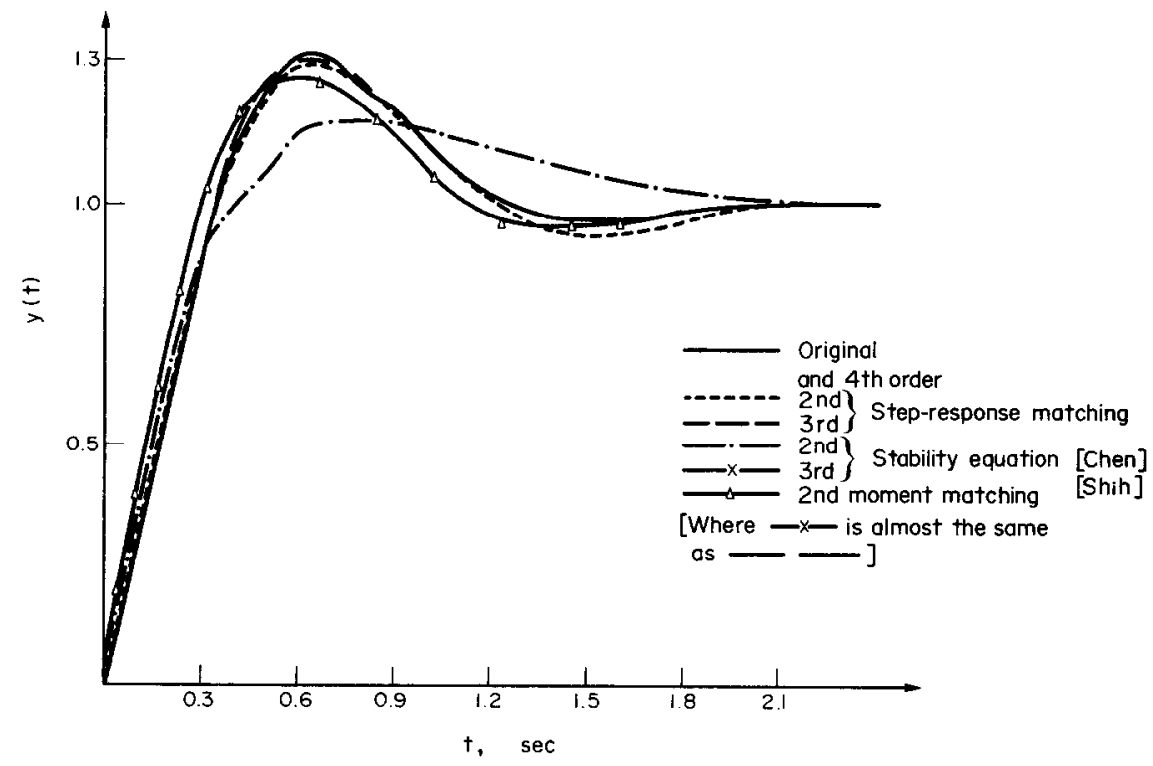

FIG. 5. Step responses of example 2.

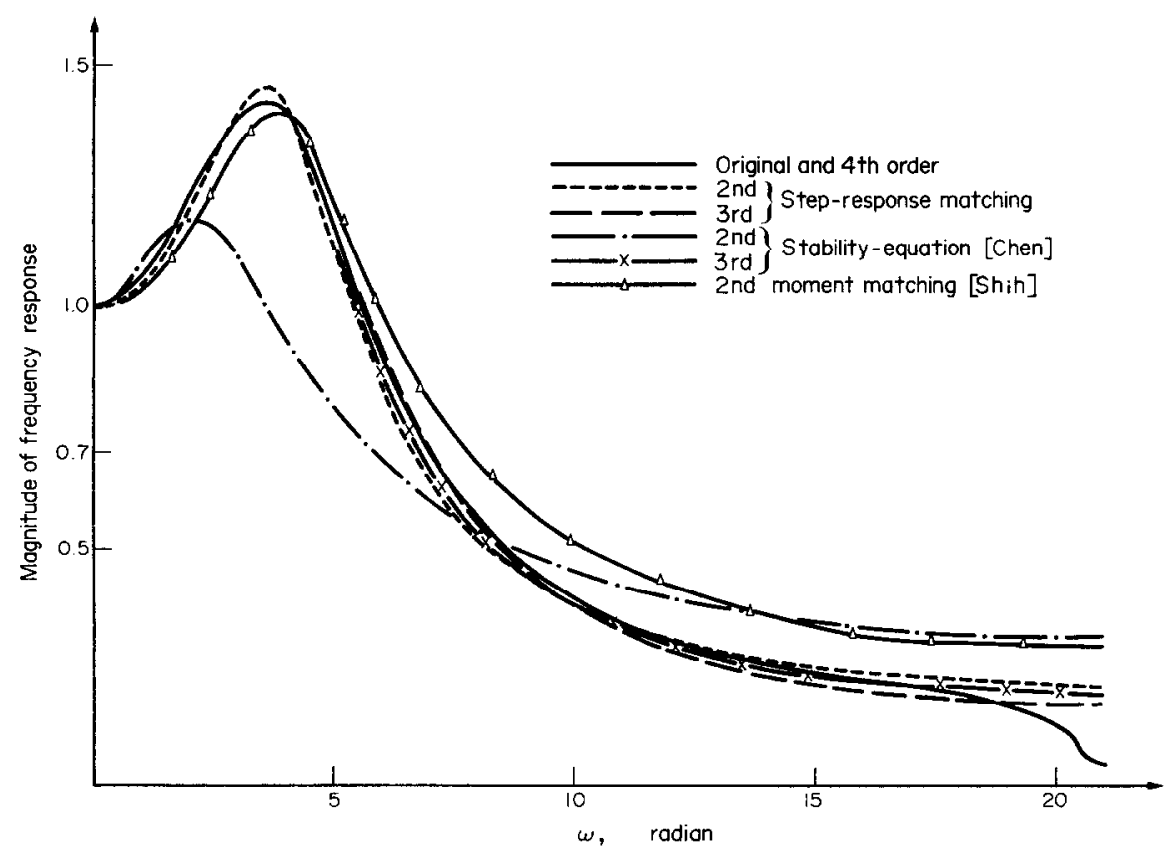

Fig. 6. Frequency responses of example 2. 
Figs. 5 and 6 , respectively. It can be seen that the reduced models based on the proposed method can give better results.

\section{Conclusions}

A method for simplification and identification of discrete transfer functions based on step-response matching have been presented. For simplification, both the step response and the frequency response of the reduced models are very close to those of the original transfer function. For identification, the proposed method has high accuracy.

\section{References}

(1) M. Decoster and A. R. Van Cauwenberghe, "A comparative study of different reduction methods", Part I and II, Journal Automatique, Vol. 17, pp. 68-74 and pp. 125-134, 1976.

(2) Y. P. Shih and W. T. Wu, "Simplification of $z$-transfer functions by continued fractions", Int. J. Control, Vol. 17, pp. 1089-1094, 1973.

(3) Y. Shamash, "Continued fraction methods for the reduction of discrete-time dynamic systems", Int. J. Control, Vol. 20, pp. 267-275, 1974.

(4) J. D. Aplevich, "Approximation of discrete linear systems", Int. J. Control, Vol. 17 , pp. $565-575,1973$.

(5) S. C. Chuang, "Homographic transformation for the simplification of discrete-time transfer functions by Pade' approximation", Int. J. Control, Vol. 22, pp. 721-728, 1975.

(6) Y. P. Shih, W. T. Wu, and H. C. Chow, "Moments of discrete systems and application in model reduction", The Chemical Engng. J., Vol. 10, pp. 107-111, 1975.

(7) T. C. Chen, C. Y. Chang, and K. W. Han, "Reduction of transfer function by the stability-equation method", J. Franklin Inst., Vol. 308, pp. 389-404, 1979.

(8) T. F. Edgar, "Least squares model reduction using step response", Int. J. Control, Vol. 22, pp. 261-270, 1975.

(9) R. H. Breen and G. C. Temes, "Application of Golub's algorithm in circuit optimization and analysis", IEEE Trans. on Circuit Theory, Vol. CT-20, pp. 687-690, 1973.

(10) I. C. Kao, "Modification of Golub's algorithm for circuit optimization", Ph.D. dissertation, Southern Methodist Univ., 1975.

(11) A. S. Householder, "Unitary triangularization of a nonsymmetric matrix", $J$. ACM, Vol. 5, pp. 339-342, 1958.

(12) J. H. Wilkinson, "Householder's method for the solution of the algebraic eigenproblem," Computer Journal, Vol. 3, pp. 23-27, 1960.

(13) K. S. Ratton and H. H. Yeh, "Discretizing continuous-data control systems," Computer-Aided Design, Vol. 10, pp. 299-306, 1978. 